\title{
Abordagens da terapia nutricional em pacientes com COVID-19: uma revisão integrativa
}

\author{
Approaches to nutritional management in patients with COVID-19: an integrative review \\ Enfoques del manejo nutricional en pacientes con COVID-19: una revisión integradora
}

Recebido: 23/10/2021 | Revisado: 31/10/2021 | Aceito: 04/11/2021 | Publicado: 07/11/2021

Flaviane Dantas de Araújo

ORCID: https://orcid.org/0000-0003-2142-7372

Universidade Federal do Rio Grande do Norte, Brasil

E-mail: flaviane.dantas.105@ufrn.edu.br

Albenize de Azevêdo Soares

ORCID: https://orcid.org/0000-0002-3008-0043

Universidade Federal do Rio Grande do Norte, Brasil

E-mail: nize.azevedo@hotmail.com

Jayara Mikarla de Lira

ORCID: https://orcid.org/0000-0002-1707-0983 Universidade Federal do Rio Grande do Norte, Brasil

E-mail: jayara-mikarla@hotmail.com

Roseane Morais da Silva

ORCID: https://orcid.org/0000-0003-4260-5553

Universidade Federal do Rio Grande do Norte, Brasil

E-mail: roseane.morais@outlook.com.br

Rayssa da Silva Felinto

ORCID: https://orcid.org/0000-0003-0191-5539

Universidade Federal do Rio Grande do Norte, Brasil

E-mail: rayssafelinto@ outlook.com

Brenda Kelly Pontes Soares

ORCID: https://orcid.org/0000-0001-7873-1653

Universidade Federal do Rio Grande do Norte, Brasil

E-mail: brendaa.pontes@gmail.com

Ana Caroline da Fonseca Nunes

ORCID: https://orcid.org/0000-0002-8445-8873

Universidade Federal do Rio Grande do Norte, Brasil

E-mail: carolfonsecanunes2000@gmail.com

Mayara Gabriela Cândido de Oliveira

ORCID: https://orcid.org/0000-0002-9669-5999

Universidade Federal do Rio Grande do Norte, Brasil

E-mail: mayaragb4@hotmail.com

Lisandra Mikaely Barboza da Silva

ORCID: https://orcid.org/0000-0002-1862-7990

Universidade Federal do Rio Grande do Norte, Brasil

E-mail: lisbarboza@outlook.com

Lorena dos Santos Tinôco

ORCID: https://orcid.org/0000-0001-6511-3522

Hospital Pediátrico Maria Alice Fernandes, Brasil

E-mail: lorena_tinoco@yahoo.com.br

José Adailton da Silva

ORCID: https://orcid.org/0000-0002-6037-7649

Departamento de Saúde Coletiva, Brasil

E-mail: silva.adaiton@ufrn.edu.br

Heleni Aires Clemente

ORCID: https://orcid.org/0000-0002-2180-6754

Universidade Federal do Rio Grande do Norte, Brasil

E-mail: heleni.aires22@hotmail.com

\section{Resumo}

O objetivo desse estudo foi realizar um levantamento na literatura cientifica das possíveis terapias nutricionais abordadas em pacientes com covid-19. É uma revisão do tipo integrativa, no qual foi realizada as buscas nas bases de dados PubMed, Scopus e Web of Science, sendo incluídos apenas artigos científicos publicados nos anos de 2020 a 2021. Para chave de busca foram considerados os descritores: "Nutrition Therapy"; "Coronavirus Infections" e sinonímias em inglês, onde foram encontrados 1.152 estudos, dos quais 8 foram incluídos. Os artigos analisados 
apresentaram diferentes condutas nutricionais; como resultados observou-se o suporte nutricional precoce de progressão lenta como alternativa eficiente, assim como, a terapia da nutrição enteral com início nas primeiras $24 \mathrm{~h}$ ou 48h de admissão na UTI. Nada obstante, o risco nutricional é um fator relevante nestes pacientes, no qual a desnutrição se faz presente na maioria dos casos, todavia, manter o estado nutricional adequado é uma prioridade para sobrevivência dos mesmos. Desta forma, o manejo nutricional adequado é fundamental para pacientes infectados por covid-19, no qual um suporte adequado com nutrição enteral e a suplementação com o ômega-3 são favoráveis à recuperação e sobrevida desses pacientes, no entanto, são necessários mais estudos para esta última afirmação.

Palavras-chave: COVID-19; Terapia nutricional; Recomendações nutricionais.

\begin{abstract}
The aim of this study was to conduct a survey in the scientific literature of possible nutritional therapies addressed in patients with covid-19. It is an integrative review, in which searches were performed in the PubMed, Scopus and Web of Science databases, including only scientific articles published in the years 2020 to 2021. For the search key, the following descriptors were considered: "Nutrition Therapy "; "Coronavirus Infections" and synonyms in English, where 1,152 studies were found, of which 8 were included. The articles analyzed presented different nutritional behaviors; as a result, early nutritional support with slow progression was observed as an efficient alternative, as well as enteral nutrition therapy starting within the first $24 \mathrm{~h}$ or $48 \mathrm{~h}$ of admission to the ICU. Nevertheless, nutritional risk is a relevant factor in these patients, in which malnutrition is present in most cases, however, maintaining an adequate nutritional status is a priority for their survival. Thus, adequate nutritional management is essential for patients infected with covid-19, in which adequate support with enteral nutrition and supplementation with omega-3 are favorable for the recovery and survival of these patients, however, more studies are needed to this last statement.
\end{abstract}

Keywords: COVID-19; Nutrition therapy; Recommended dietary allowances.

\title{
Resumen
}

El objetivo de este estudio fue realizar una encuesta en la literatura científica de posibles terapias nutricionales dirigidas a pacientes con covid-19. Se trata de una revisión integradora, en la que se realizaron búsquedas en las bases de datos PubMed, Scopus y Web of Science, incluyendo solo artículos científicos publicados en los años 2020 a 2021. Para la clave de búsqueda se consideraron los siguientes descriptores: "Nutrition Therapy"; "Infecciones por coronavirus" y sinónimos en inglés, donde se encontraron 1,152 estudios, de los cuales 8 fueron incluidos. Los artículos analizados presentaron diferentes comportamientos nutricionales; como resultado, se observó como una alternativa eficaz el soporte nutricional precoz y de progresión lenta, así como la terapia de nutrición enteral iniciada dentro de las primeras 24 o $48 \mathrm{~h}$ del ingreso en UCI. Sin embargo, el riesgo nutricional es un factor relevante en estos pacientes, en los cuales la desnutrición está presente en la mayoría de los casos, sin embargo, mantener un estado nutricional adecuado es una prioridad para su supervivencia. Así, un adecuado manejo nutricional es fundamental para los pacientes infectados por covid-19, en los que un adecuado soporte con nutrición enteral y suplementación con omega-3 son favorables para la recuperación y supervivencia de estos pacientes, sin embargo, se necesitan más estudios a esta última afirmación.

Palabras clave: COVID-19; Terapia nutricional; Ingesta diaria recomendada.

\section{Introdução}

A COVID-19 é uma doença infecciosa causada pelo novo coronavírus, também denominada síndrome respiratória aguda grave 2 (SARS-Cov-2), alvo principal do desencadeando de uma pandemia no ano de 2020, permanecendo até o momento atual (Patel et al., 2020). Sua contaminação se dá através de gotículas no ar por pessoas infectadas - assintomáticas ou não, com risco de transmissão presente, cujo os sintomas mais comuns são tosse, febre, secreções nasais, dispneia, mialgia, e em casos mais graves, apresentam dificuldade de respiração após o período médio de incubação (3-7 dias), com variação de 2 - 14 dias (Grupta et al., 2020; Gorbalenya et al., 2020).

Os estágios da doença podem ser classificados em 3, sendo eles: estágio 1 (infecção inicial), no qual o vírus se multiplica e começa a afetar inicialmente os pulmões, apresentando sinais e sintomas leves a moderados, como febre, tosse seca, pneumonia e diarreia; estágio 2 (fase pulmonar) é caracterizada pela hipóxia, falta de ar e sinais de imagem torácica anormal; e o estágio 3 (fase de hiperinflamação) apresenta sinais de marcadores inflamatórios elevados (IL-6, ferritina, troponina, dímero D), e caracteriza em sintomas clínicos de síndrome do desconforto respiratório agudo, insuficiência cardíaca, disfunção de múltiplos órgãos e síndrome da resposta inflamatória sistêmica. Os dois últimos estágios são marcados por respostas inflamatórias altas, levando a tempestade de citocinas e morte não controlada, por este motivo, a depender da 
gravidade da doença, disseminação da infecção e carga viral é que é decidido o ambiente de tratamento (Grupta et al., 2020).

Estudos demostram que há um número significativo de pacientes que requerem hospitalização, dos quais alguns necessitam da unidade de terapia intensiva (UTI) com apoio multiprofissional. Dentre estes indivíduos, os que estão em condições criticamente graves por covid-19, encontra-se com o estado nutricional comprometido, catabólico e com elevada inflamação no organismo, levando assim, a condição de desnutrição com significativa taxa mortalidade. Desta forma, se faz necessário avaliá-los de acordo com as recomendações especificas, visando uma oferta energética lenta para segurança do paciente e evitando uma possível síndrome de realimentação, no entanto, a maioria dos indivíduos precisam de ventilação mecânica, o que tornam impossibilitados de alimentar-se por via oral, por este motivo, demanda de uma nutrição enteral e/ou parenteral (Ferreira et al.,2020; Wu \& McGoogan, 2020).

A pandemia do COVID-19 desafiou os profissionais de saúde a executar condutas diante uma doença antes desconhecida, além de reforçar a necessidade de mais evidências cientificas em que fosse possível embasar o fortalecimento do sistema imunológico e terapias de suporte de UTI (Morreira et al., 2020). Desta forma, o manejo adequado é ponto essencial para fornecer uma intervenção nutricional assertiva, como meio de contribuição para um desfecho favorável da doença. Assim, desse estudo foi realizar um levantamento na literatura cientifica das possíveis terapias nutricionais abordadas em pacientes com covid-19.

\section{Metodologia}

Trata-se de uma pesquisa que utilizou como método a revisão de literatura do tipo integrativa, a qual abrange seis fases para a sua elaboração: a identificação do tema e elaboração da questão de pesquisa, busca na literatura, coleta de dados, análise crítica dos estudos incluídos, interpretação e discussão dos resultados e apresentação da síntese de conhecimento. Desse modo, permite reunir resultados de pesquisas, de forma sistemática e ordenada, contribuindo com o aprofundamento do conhecimento do tema investigado (Sousa et al., 2017; Soares et al., 2014).

Para direcionamento deste estudo, foi elaborada a seguinte questão de pesquisa: "Quais as evidências científicas sobre o manejo nutricional de pacientes com COVID-19?". Na construção de uma questão norteadora adequada, utilizou-se a estratégia PICO: "P" corresponde à população (Pacientes com COVID-19); "I" à intervenção (Terapia Nutricional); "C" à comparação (não aplicável neste estudo) e "O" ao desfecho (Efeito do manejo nutricional nos pacientes com Covid-19) (Santos et al., 2007).

O levantamento das publicações ocorreu no período de junho e julho de 2021 nas bases de dados PubMed, Scopus e Web of Science sendo estabelecidos limites quanto ao ano de publicação de 2020 a 2021. Com a finalidade de garantir a busca ampla, fez-se uso do portal de periódicos da Coordenação de Aperfeiçoamento de Pessoal de Nível Superior (CAPES), com reconhecimento na Universidade Federal do Rio Grande do Norte (UFRN).

Para o refinamento dos artigos estabeleceram-se como critérios de inclusão: artigos publicados em português, espanhol e inglês e que atendessem ao objetivo da pesquisa, publicados no período de ano anteriormente referido. Foram excluídos editoriais, cartas ao editor, resumos, opinião de especialistas, outras revisões, correspondências, resenhas, capítulos de livros, teses, dissertações, artigos repetidos e que não atendesse o objetivo proposto.

Para busca foram selecionados os descritores controlados do vocabulário descritores em Ciência da Saúde (DECs), Medical Subject Headings (MESH), e EMBASE Emtreee na língua inglesa: Nutrition Therapy; Coronavirus Infections e sinonímias em inglês. O cruzamento desses descritores ocorreu por meio do operador booleano AND (Tabela 1): 
Tabela 1 - Cruzamento dos descritores utilizados. Santa Cruz, Rio Grande do Norte, Brasil, 2021.

Cruzamento:

("Nutrition Therapy" OR "Therapy, Nutrition" OR "Medical Nutrition Therapy" OR "Nutrition Therapy, Medical" OR "Therapy, Medical Nutrition" OR "diet therapy" OR "diet intervention" OR "diet treatment" OR "dietary intervention" OR "dietary therapy" OR "dietary treatment" OR "nutritional therapy") AND ("Coronavirus Infections" OR "Coronavirus Infection" OR "COVID-19” OR "2019 novel coronavirus disease" OR "COVID-19 pandemic" OR "SARS-CoV-2 infection" OR "COVID-19 virus disease" OR "2019 novel coronavirus infection" OR "2019-nCoV infection" OR "Coronavirus disease 2019" OR "Coronavirus disease-19” OR "2019-nCoV disease" OR "COVID-19 virus infection")

Fonte: Autores (2021).

A Tabela 2 apresenta os resultados das buscas a partir das bases elegidas:

Tabela 2 - Resultados nas bases de dados elegidas. Santa Cruz, Rio Grande do Norte, Brasil, 2021.

\begin{tabular}{|c|c|c|c|c|}
\hline Bases de Dados & Artigos Encontrados & Artigos Excluídos & Artigos Analisados & Artigos Incluídos \\
\hline PubMed & 853 & 844 & 9 & 2 \\
\hline SCOPUS & 235 & 213 & 22 & 5 \\
\hline Web of Science & 64 & 53 & 11 & 1 \\
\hline
\end{tabular}

Fonte: Autores (2021).

O Fluxograma 1 apresenta o processo de elaboração da revisão:

Fluxograma 1 - Processo de seleção, elegibilidade e inclusão dos artigos na revisão integrativa. Santa Cruz, Rio Grande do Norte, Brasil, 2021.
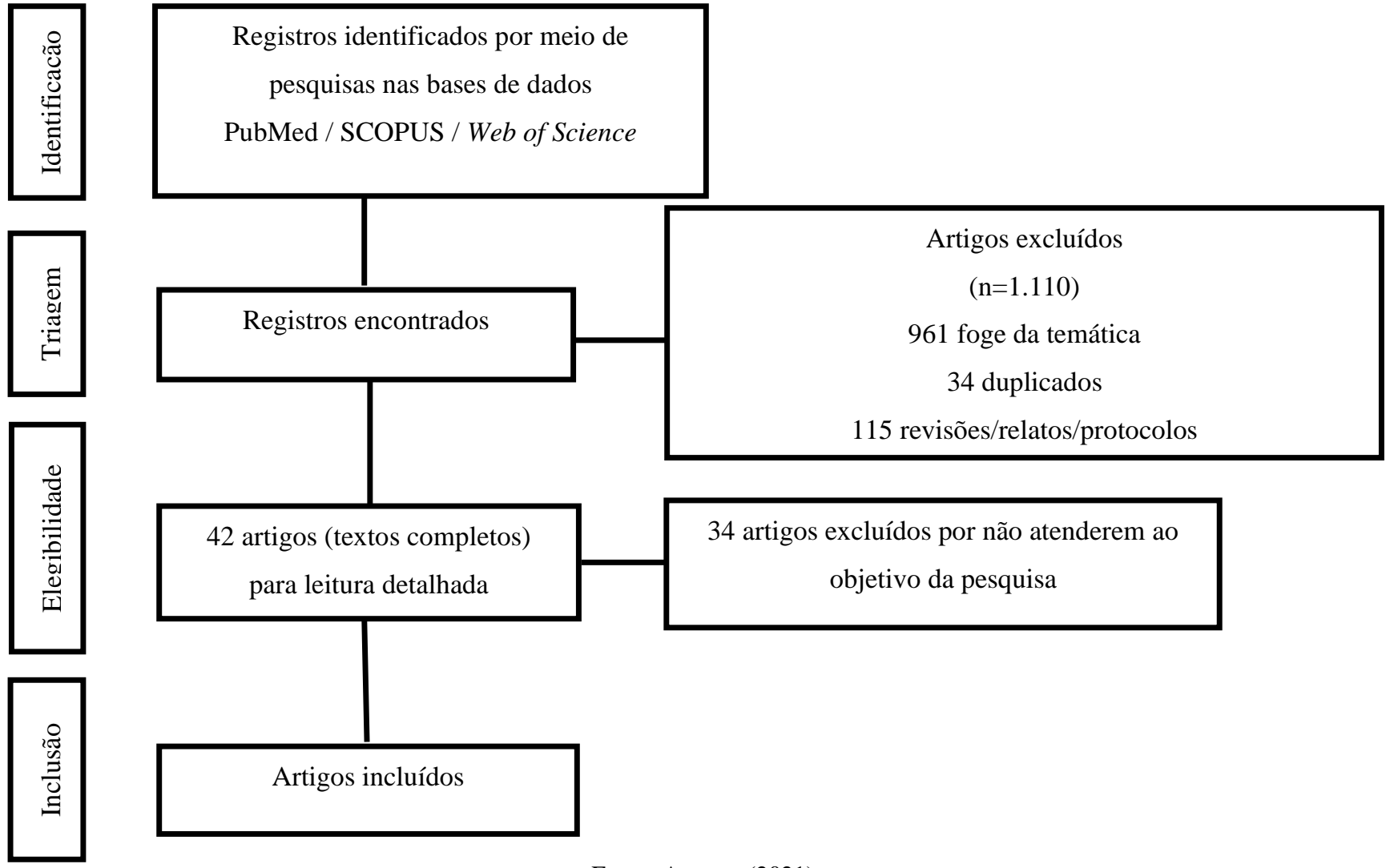

Fonte: Autores (2021). 


\section{Resultados e Discussão}

A amostra foi composta por 8 artigos selecionados para a análise final, nos quais foram publicados nos seguintes periódicos de jornais científicos: (3) Jornal Nutrition in Clinical Practice, (2) Jornal Clinical Nutrition ESPEN, (1) Journal Clinical Nutrition, (1) Jornal of Parenteral and enteral Nutrition, (1) Jornal of Translation Medicine.

Os artigos apontaram a caracterização de pacientes diagnosticados com COVID-19, onde (4) estudos abordaram pacientes graves com COVID-19, (2) pacientes em ventilação mecânica por COVID-19, (2) pacientes diagnosticados com COVID-19. Os locais nos quais ocorreram às pesquisas foram: Unidades de Terapias Intensivas (UTI) (6) e Clinicas médicas de hospitais (2). Na caracterização metodológica e delineamento da pesquisa dos artigos analisados, detectou-se que a maioria utilizou os estudos de coorte, onde (4) eram do tipo ensaio de coorte, (3) do tipo observacional e (1) ensaio clínico randomizado.

A Tabela 3 apresenta a síntese dos artigos selecionados para a revisão integrativa da literatura.

Tabela 3 - Síntese dos artigos selecionados para a revisão integrativa da literatura. Santa Cruz, Rio Grande do Norte, Brasil, 2020.

\begin{tabular}{|c|c|c|c|c|c|}
\hline $\mathbf{N}^{\circ}$ & Título & Autor/ Base de dados & Periódico / Ano & Objetivo & Síntese dos resultados \\
\hline 1 & $\begin{array}{l}\text { Safety and tolerance of } \\
\text { enteral nutrition in } \\
\text { COVID-19 critically ill } \\
\text { patients, a retrospective } \\
\text { study }\end{array}$ & $\begin{array}{c}\text { Iván Osuna-Padilla; } \\
\text { Nadia Carolina } \\
\text { Rodríguez-Moguel; } \\
\text { Adriana Aguilar- } \\
\text { Vargas; Sebastián } \\
\text { Rodríguez-Llamazares } \\
\text { / Scopus }\end{array}$ & $\begin{array}{c}\text { Clinical } \\
\text { Nutrition } \\
\text { ESPEN / } 2021\end{array}$ & $\begin{array}{l}\text { Analisar a incidência } \\
\text { de intolerância } \\
\text { gastrointestinal } \\
\text { associada à Nutrição } \\
\text { enteral (diarreia, } \\
\text { vômitos, gastroparesia } \\
\text { e constipação) na } \\
\text { primeira semana, e } \\
\text { avaliar associações } \\
\text { entre dados clínicos e } \\
\text { laboratoriais com } \\
\text { intolerância GI e } \\
\text { déficits energético- } \\
\text { protéicos em pacientes } \\
\text { críticos pacientes } \\
\text { recebendo ventilação } \\
\text { mecânica devido a } \\
\text { COVID-19 } \\
\text { confirmado. }\end{array}$ & $\begin{array}{c}\text { A nutrição enteral é uma } \\
\text { intervenção segura, viável e } \\
\text { tolerável para pacientes graves } \\
\text { de COVID-19 sob ventilação } \\
\text { mecânica na primeira semana } \\
\text { de intubação. } \\
90 \% \text { dos pacientes que } \\
\text { receberam essa terapia } \\
\text { alcançaram } 80 \% \text { da meta de } \\
\text { necessidades no } 7^{\circ} \text { dia, } \\
\text { tornando possível descartar } \\
\text { qualquer preocupação clinica } \\
\text { comum de intolerância } \\
\text { gastrointestinal nestes, além } \\
\text { do mais, o diagnóstico de } \\
\text { Lesão renal aguda foi } \\
\text { associado ao maior déficit de } \\
\text { energia no } 7^{\circ} \text { dia; a } \\
\text { hipernatremia foi uma das } \\
\text { alterações eletrolíticas mais } \\
\text { frequentes. }\end{array}$ \\
\hline 2 & $\begin{array}{l}\text { Malnutrition and } \\
\text { nutritional therapy in } \\
\text { patients with SARS-CoV- } \\
2 \text { disease }\end{array}$ & $\begin{array}{l}\text { Loris Pironi; Anna } \\
\text { Simona Sasdellia; } \\
\text { Federico Ravaioli; } \\
\text { Bianca Baracco; } \\
\text { Claudia Battaiola; } \\
\text { Giulia Bocedia; Lucia } \\
\text { Brodosia; Laura Leoni; } \\
\text { Giulia Aurora Mari; } \\
\text { Alessandra Musio } \\
\text { / Scopus }\end{array}$ & $\begin{array}{c}\text { Clinical } \\
\text { nutrition / } 2021\end{array}$ & $\begin{array}{c}\text { Conhecer a } \\
\text { prevalência de } \\
\text { desnutrição, bem } \\
\text { como, a terapia } \\
\text { nutricional fornecida a } \\
\text { adultos com COVID- } \\
19 \text { internados em um } \\
\text { hospital de terceiro } \\
\text { nível na Itália, por } \\
\text { meio de uma auditoria. }\end{array}$ & $\begin{array}{l}\text { Prevalência muito elevada de } \\
\text { risco nutricional }(77,2 \%) \text { e } \\
\text { desnutrição }(49,7 \%) \text {, em que, } \\
\text { a terapia nutricional mostrou- } \\
\text { se no limite inferior ou abaixo } \\
\text { das recomendações. Os } \\
\text { pacientes que estavam com } \\
\text { dieta oral foram afetados } \\
\text { adversamente devido o } \\
\text { comprometimento do apetite, } \\
\text { fragilidade e processo } \\
\text { invasivo do O } \text { Ond todavia, sendo }_{\text {mais frequente nos da UTI. }} \\
\text { No entanto, os internados em } \\
\text { UTI apresentaram ingestões } \\
\text { significativas menores quando } \\
\text { comparados aos que não } \\
\text { estavam neste ambiente. }\end{array}$ \\
\hline
\end{tabular}




\begin{tabular}{|c|c|c|c|c|c|}
\hline 3 & $\begin{array}{c}\text { Association Between Low } \\
\text { Zinc Levels and Severity } \\
\text { of Acute Respiratory } \\
\text { Distress Syndrome by } \\
\text { New Coronavirus SARS- } \\
\text { CoV-2 }\end{array}$ & $\begin{array}{c}\text { Dr. Thiago Jose } \\
\text { Martins Gonçalves; } \\
\text { Médica Sandra Elisa } \\
\text { Adami Batista } \\
\text { Gonçalves; Andreia } \\
\text { Guarnieri MD; } \\
\text { Rodrigo Cristovão } \\
\text { Risegato MD; Maysa } \\
\text { Penteado Guimarães } \\
\text { MD; Daniella Cabral } \\
\text { de Freitas MD; Alvaro } \\
\text { Razuk-Filho MD; } \\
\text { PhD; Pedro Batista } \\
\text { Benedito Junior MD; } \\
\text { Eduardo Fagundes } \\
\text { Parrillo MD / Scopus }\end{array}$ & $\begin{array}{c}\text { Nutrition in } \\
\text { Clinical Practice } \\
\text { / } 2021\end{array}$ & $\begin{array}{l}\text { Avaliar a prevalência } \\
\text { de baixos níveis de } \\
\text { zinco em pacientes } \\
\text { gravemente enfermos } \\
\text { infectados pela SARS- } \\
\text { CoV-2 e sua } \\
\text { associação com a } \\
\text { gravidade da síndrome } \\
\text { do desconforto } \\
\text { respiratório agudo } \\
\text { (SDRA). }\end{array}$ & $\begin{array}{l}\text { Baixos níveis de zinco foram } \\
\text { encontrados em pacientes } \\
\text { gravemente enfermos } \\
\text { infectados pela SARS-CoV-2 } \\
(79,6 \%) \text {, sendo muito } \\
\text { prevalente na admissão à UTI. } \\
\text { Ainda assim, estes } \\
\text { apresentaram associação com } \\
\text { grandes chances de } \\
\text { diagnóstico de SDRA. }\end{array}$ \\
\hline 4 & $\begin{array}{c}\text { Influence of nutritional } \\
\text { assistance on mortality by } \\
\text { COVID-19 in critically ill } \\
\text { patients }\end{array}$ & $\begin{array}{l}\text { Thaisy Cristina } \\
\text { Honorato Santos } \\
\text { Alves; Renata Santos } \\
\text { Guimarães; Sineide } \\
\text { Freitas de Souza; } \\
\text { Najara Amaral } \\
\text { Brandão; Carla Hilário } \\
\text { da Cunha Daltro; } \\
\text { Maria Ester Pereira } \\
\text { Conceição-Machado; } \\
\text { Lucivalda Pereira } \\
\text { Magalhães de Oliveira; } \\
\text { Carla de Magalhães } \\
\text { Cunha / Scopus }\end{array}$ & $\begin{array}{c}\text { Clinical } \\
\text { Nutrition } \\
\text { ESPEN / } 2021\end{array}$ & $\begin{array}{l}\text { Avaliar a influência da } \\
\text { assistência nutricional } \\
\text { na mortalidade do } \\
\text { COVID-19 em } \\
\text { pacientes internados } \\
\text { em unidades de terapia } \\
\text { intensiva (UTI). }\end{array}$ & $\begin{array}{l}\text { A terapia nutricional com } \\
\text { inicio } 48 \text { horas após a } \\
\text { admissão em associação com } \\
\text { a presença de obesidade é } \\
\text { considerada fator de risco para } \\
\text { mortalidade pelo COVID-19. } \\
\text { Diante disso, a implantação do } \\
\text { manejo nutricional adequado } \\
\text { visa minimizar o } \\
\text { comprometimento do estado } \\
\text { nutricional e favorecer uma } \\
\text { recuperação clínica. }\end{array}$ \\
\hline 5 & $\begin{array}{l}\text { Nutrition evaluation and } \\
\text { management of critically } \\
\text { ill patients with COVID- } \\
19 \text { during post-intensive } \\
\text { care rehabilitation }\end{array}$ & $\begin{array}{c}\text { Alice Hoyois; } \\
\text { Asuncion Ballarin; } \\
\text { Justine Thomas; } \\
\text { Olivier Lheureux; } \\
\text { Jean-Charles Preiser; } \\
\text { Emmanuel Coppens; } \\
\text { Silvia Perez-Bogerd; } \\
\text { Olivier Taton; Sylvie } \\
\text { Farine; Pauline Van } \\
\text { Ouytsel; Marianna } \\
\text { Arvanitakis / Scopus }\end{array}$ & $\begin{array}{c}\text { Journal of } \\
\text { Parenteral and } \\
\text { Enteral } \\
\text { Nutrition / } 2021\end{array}$ & $\begin{array}{l}\text { Avaliar o estado } \\
\text { nutricional e o } \\
\text { resultado da terapia } \\
\text { nutricional em } \\
\text { pacientes com } \\
\text { COVID-19 após a alta } \\
\text { da UTI. }\end{array}$ & $\begin{array}{c}\text { A desnutrição grave e a baixa } \\
\text { massa muscular estavam } \\
\text { presentes na maioria dos } \\
\text { pacientes pós alta da UTI com } \\
\text { ventilação mecânica. } \\
\text { Ademais, com a terapia } \\
\text { nutricional de } \\
\text { acompanhamento - Inicial: } \\
\text { NE ou NP + oral (NP em } \\
\text { exceção de } 1 \text { paciente) Final: } \\
\text { Oral isolada em associação } \\
\text { com a reabilitação física foi } \\
\text { possível visualizar uma } \\
\text { melhora significativa dos } \\
\text { parâmetros nutricionais, no } \\
\text { qual é necessário suporte } \\
\text { crucial de ingestão adequada } \\
\text { de proteínas (>1,5 g/kg/dia) } \\
\text { após alta hospitalar. }\end{array}$ \\
\hline 6 & $\begin{array}{l}\text { Early Enteral Nutrition in } \\
\text { Mechanically Ventilated } \\
\text { Patients } \\
\text { With COVID-19 Infection }\end{array}$ & $\begin{array}{l}\text { Nicholas Farina; Sarah } \\
\text { Nordbeck; } \\
\text { Michelle Montgomery; } \\
\text { Laura Cordwin; Faith } \\
\text { Blair; } \\
\text { Jill Cherry-Bukowiec; } \\
\text { Michael D. Kraft; } \\
\text { Melissa R. Pleva; Erica } \\
\text { Raymond / Web of } \\
\text { science }\end{array}$ & $\begin{array}{l}\text { Nutrition in } \\
\text { Clinical } \\
\text { Practice/ } 2021\end{array}$ & $\begin{array}{c}\text { Associar a provisão } \\
\text { de nutrição enteral } \\
\text { precoce com } \\
\text { resultados em } \\
\text { pacientes ventilados } \\
\text { mecanicamente com } \\
\text { COVID-19 e } \\
\text { secundariamente } \\
\text { descrever práticas } \\
\text { nutricionais em } \\
\text { pacientes com } \\
\text { ventilação mecânica } \\
\text { tratados com COVID- } \\
19 .\end{array}$ & $\begin{array}{c}\text { Pacientes }(\mathrm{n}=60) \text { que } \\
\text { receberam nutrição enteral em } \\
24 \text { horas do início da } \\
\text { ventilação mecânica recebeu } \\
\text { significativamente mais } \\
\text { calorias e proteína } \\
\text { diariamente. Além de } \\
\text { pontuações no APACHE II e } \\
\text { SOFA serem } \\
\text { significativamente menores. } \\
\text { Iniciar a nutrição enteral em } \\
24 \text { horas de ventilação } \\
\text { mecânica não foi associada a } \\
\text { uma diferença no tempo de } \\
\text { internação, mortalidade de } \\
\text { pacientes internados, dias }\end{array}$ \\
\hline
\end{tabular}


hipofosfatemia grave.

7 The efect of omega-3 fatty acid supplementation on clinical and biochemical parameters of critically ill patients with COVID-19: a randomized clinical trial

\author{
Saeid Doaei, Somayeh \\ Gholami, Samira \\ Rastgoo, Maryam \\ Gholamalizadeh, \\ Fatemeh Bourbour, \\ Seyedeh Elaheh \\ Bagheri, Forough \\ Samipoor, Mohammad \\ Esmail Akbari, Mahdi \\ Shadnoush, Fereshteh \\ Ghorat, Seyed Alireza \\ Mosavi Jarrahi, Narjes \\ Ashouri Mirsadeghi, \\ Azadeh Hajipour, \\ Parvin Joola, Alireza \\ Moslem and Mark \\ O.Goodarzi / Pubmed
}

Journal of
Translational
Medicine / 2021

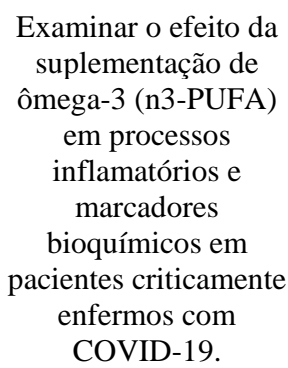

Examinar o efeito da suplementação de ômega-3 (n3-PUFA) em processos inflamatórios e marcadores bioquímicos em pacientes criticamente enfermos com COVID-19.

101 pacientes foram incluídos na análise, dos quais 28 foram atribuídos ao grupo de tratamento e 73 foram atribuídos ao grupo de controle. A administração de ômega-3 melhorou significativamente o $\mathrm{PH}$ arterial, o $\mathrm{HCO} 3$, a $\mathrm{Be}$, os níveis dos indicadores da função renal, incluindo BUN,Cr, K e o volume da urina, como também pode aumentar a contagem de linfócitos.O grupo de intervenção teve uma taxa de sobrevivência significativamente maior, em torno de 1 mês em comparação com o grupo de controle

\begin{tabular}{|c|c|c|c|c|c|}
\hline 8 & $\begin{array}{l}\text { Nutrition Support in } \\
\text { Patients With Acute } \\
\text { Respiratory Distress } \\
\text { Syndrome COVID-19 }\end{array}$ & $\begin{array}{c}\text { Paolo Formenti, MD / } \\
\text { Pubmed }\end{array}$ & $\begin{array}{c}\text { Nutrition in } \\
\text { Clinical Practice } \\
\text { / } 2021\end{array}$ & $\begin{array}{c}\text { Fornecer } \\
\text { recomendações sobre } \\
\text { uma ideal ingesta } \\
\text { calórica e protéica de } \\
\text { acordo com o } \\
\text { protocolo europeu de } \\
\text { nutrição aos pacientes } \\
\text { internados na UTI } \\
\text { diagnosticados com } \\
\text { covid-19. }\end{array}$ & $\begin{array}{l}60 \text { pacientes internados na } \\
\text { UTI com diagnóstico para } \\
\text { covid-19 e com balanço de } \\
\text { nitrogênio negativo foram } \\
\text { admitidos no estudo, onde } \\
\text { foram submetidos a um } \\
\text { aumento da ingesta calórica e } \\
\text { protéica de acordo com as } \\
\text { recomendações do protocolo } \\
\text { europeu de nutrição. Os } \\
\text { resultados mostraram que } \\
\text { ouve o alcance das metas } \\
\text { nutricionais, entretanto o } \\
\text { balanço de nitrogênio } \\
\text { permaneceu negativo, } \\
\text { enfatizando a idéia de que as } \\
\text { diretrizes nutricionais } \\
\text { existentes até o momento do } \\
\text { estudo não são suficientes. }\end{array}$ \\
\hline
\end{tabular}

Fonte: Autores (2021).

A análise dos resultados se deu a partir da leitura dos estudos. Esse processo determinou o apanhado dos seguintes eixos temáticos. "Condutas nutricionais para pacientes graves com covid-19", "Presença de riscos nutricionais e desnutrição grave em pacientes com Covid-19" e "Benefícios e recomendações da nutrição enteral nos pacientes graves com Covid-19".

\section{Condutas nutricionais para pacientes graves com COVID-19}

Manter o bom estado nutricional do paciente com diagnóstico de COVID-19 é de extrema relevância tanto para o enfrentamento do vírus, como para a prevenção de novas comorbidades, pois conforme os sintomas evoluem, os riscos da deficiência nutricional aumentam, o que pode acarretar no agravamento do seu quadro clínico (Souza, 2021). 
De acordo com a Sociedade Europeia de Nutrição Enteral e Parenteral (ESPEN), todos os pacientes admitidos na unidade hospitalar por diagnóstico de COVID-19 devem ser submetidos à triagem para a identificação de possíveis riscos nutricionais. Na presença desses, a avaliação deverá ocorrer de maneira mais detalhada, visando o planejamento de medidas de monitoramento e intervenção através da implementação de uma terapia nutricional adequada as necessidades do paciente favorecendo a sua recuperação clínica (Barazzoni et al., 2020).

Recomenda-se que o inicio do suporte nutricional para pacientes graves com COVID-19 seja precoce e de progressão lenta, com frequente monitorização de sinais de intolerância digestiva e com limiar reduzido para a utilização da nutrição parenteral - caso a enteral não progrida, objetivando melhorar o quadro clínico, evitar o agravamento dos sintomas e possível necessidade de cuidados intensivos (Martindale, et al., 2014).

Outro ponto relevante abordado pelo presente estudo foi a administração da suplementação de ômega-3 nos pacientes com COVID-19, melhorando significativamente o PH arterial, o HCO3, a Be, os níveis dos indicadores da função renal incluindo BUN, Cr, K e o volume da urina, como também elevou a contagem de linfócitos, além de aumentar a taxa de sobrevivência de 1 mês nos pacientes criticamente enfermos com COVID-19 (Doaei et al., 2021). A revisão sistemática de Manzanares et al. (2014), corrobora com os achados deste estudo ao constatar que a suplementação com ômega-3 pode melhorar a sobrevida em pacientes criticamente enfermos. Por outro lado, a revisão de Koekkoek, Panteleon e Zanten (2019) chegou a conclusão que as evidências sobre os benefícios da suplementação de ômega-3 em pacientes graves hospitalizados na UTI ainda são fracas, sendo necessário novos estudos para de fato confirmar esse achado.

\section{Presença de riscos nutricionais e desnutrição grave em pacientes com COVID-19}

Os estudos mostraram uma elevada prevalência de risco nutricional e desnutrição em pacientes adultos internados com COVID-19, em que, a terapia nutricional mostrou-se no limite inferior ou abaixo das recomendações. Segundo Barazzoni et al (2020) diversos são os fatores de riscos que podem afetar o estado nutricional do paciente hospitalizado com COVID-19, como por exemplo: a inflamação sistêmica e as alterações metabólicas decorrente do agravamento da própria doença, a mobilidade reduzida e a inapetência. Dessa forma, é indispensável que esses pacientes sejam triados, diagnosticados e tratados através de métodos de avaliação nutricional eficientes para a diminuição desses riscos.

Outro achado importante foi que os pacientes que estavam em dieta oral tiveram seu estado nutricional afetado adversamente devido o comprometimento do apetite, da fragilidade e do processo invasivo do O2 (Loris et al, 2021). Em consonância a esse achado, o estudo realizado na Europapor Lechien et al, (2020) com pacientes diagnosticados com COVID19 provenientes de hospitais da Bélgica, França, Espanha e Itália mostrou que, 85,6\% dos pacientes tiveram distúrbios olfatórios e 88,8\% apresentaram distúrbios gustatórios relacionados à infecção que pode acarretar diretamente a inapetência, fazendo com que os pacientes não consigam atingir suas metas nutricionais. Desse modo, faz-se necessário utilizar formas de apresentação variadas, para favorecer a manutenção/aumento da ingestão proteico-calórica, além dos suplementos nutricionais orais.

Pode-se observar nesta revisão uma prevalência de 79,6\% de baixos níveis de zinco entre os pacientes gravemente enfermos com COVID-19 admitidos na UTI. Linko et al (2011) trouxe em seu estudo que, era comum os baixos níveis de zinco no início da insuficiência respiratória aguda em pacientes internados na UTI, entretanto, esse achado não foi preditivo para o aumento da mortalidade em 30 dias de internação nesses pacientes, como também não influenciou no tempo de internação nem na necessidade de ventilação mecânica. 


\section{Benefícios e recomendações da nutrição enteral nos pacientes graves com COVID-19}

Conforme as definições da terapia nutricional do regulamento técnico para Terapia Nutricional Enteral (TNE), a resolução RDC n.63, da Agência Nacional de Vigilância Sanitária (ANVISA) do Ministério da Saúde 6 de julho de 2000, caracterizou a NE como uma alimentação com finalidades especiais e com ingestão controlada de nutrientes na forma isolada ou combinada, formulada especialmente para uso por via oral ou por sondas, sendo usada para substituir ou complementar a alimentação oral de pacientes desnutridos ou não, de acordo com suas necessidades nutricionais para à preservação dos órgãos, sistemas e tecidos (Brasil, 2000).

A nutrição enteral (NE) é uma terapia nutricional que deverá ser iniciada quando a alimentação por via oral não for possível, sendo sugerido o início dentro de $24 \mathrm{~h}$ a $48 \mathrm{~h}$, em pacientes hemodinamicamente compensados e principalmente se precisar de intubação e ventilação mecânica. A NE é mais aconselhável do que a NP, desde que o paciente esteja com um bom funcionamento do trato gastrointestinal (Mcclave et 2020).

Pode-se observar nesse estudo que a nutrição enteral é uma intervenção segura, viável e tolerável para pacientes graves de COVID-19 sob ventilação mecânica na primeira semana de intubação, além de que, pacientes que receberam nutrição enteral em 24 horas do início da ventilação mecânica, recebeu significativamente mais calorias e proteína diariamente, sem contar que a terapia nutricional com início 48 horas após a admissão em associação com a presença de obesidade é considerada fator de risco para mortalidade pela COVID-19 (Osuna-Padilla et al., 2021; Farina et al., 2021; Alves et al., 2021 ). Conforme Singer et al, (2019) "As diretrizes da ESPEN, ASPEN e SCCM mostram que através de pesquisas com ensaios randomizados clínicos, pode-se concluir que o fornecimento de NE precoce para pacientes intervencionistas melhorou a mortalidade e reduziu infecções em comparação com os casos no qual essa terapia foi adiada ou suspensa."

\section{Conclusão}

A terapia nutricional em pacientes com a infecção pelo novo coronavírus (SARS-Cov-2) apresenta-se como uma intervenção fundamental para um bom enfrentamento da doença COVID-19, além de prevenir o agravamento das comorbidades, evita o risco nutricional e desenvolvimento da desnutrição, ocasionada muitas vezes devido à fragilidade na realização da triagem nutricional no momento da admissão e durante condutas nutricionais fragilmente fundamentadas.

Além disso, a divergência dos estudos leva a falta de um cuidado com práticas mais seguras e com garantias cientificas de bons resultados nos pacientes enfermos pela COVID-19. Nesse sentido, observa-se que o início precoce do manejo nutricional já é uma certeza de bons resultados segundo a literatura analisada, principalmente no intervalo de 24 a 48 horas. No entanto, há segurança sobre como fazer? O que avaliar ao longo das terapias nutricionais? E quais nutrientes estimular? Ainda é uma zona cinzenta diante das evidencias estudadas.

Nesse cenário, a terapia enteral destaca-se como melhor suporte nutricional enteral e a suplementação com o ômega-3 aparecem como favorável a recuperação e sobrevida desses pacientes. Entretanto, constata-se, neste estudo, que as recomendações são atualizadas constantemente, à proporção que as evidências indicam a necessidade de novas ações, o que demanda dos profissionais da saúde sucessivas atualizações.

Dessa forma, faz-se necessário o desenvolvimento de estudos futuros que abarquem condutas eficientes para o manejo nutricional de acordo com o estado clinico do paciente, além de alternativas alimentares para os pacientes em cuidados domiciliares e hospitalares contaminados com o COVID-19. Ademais, evidencias cientificas permitem um cuidado nutricional eficaz, com a finalidade de prevenir a morbimortalidade em grande proporção ocasionada pelo Coronavírus. 


\section{Referências}

Alves, T. C. H. S., Guimarães, R. S., de Souza, S. F., Brandão, N. A., da Cunha Daltro, C. H., Conceição-Machado, M. E. P., \& de Magalhães Cunha, C. (2021). Influence of nutritional assistance on mortality by COVID-19 in critically ill patients. Clinical Nutrition ESPEN. 44, 469-471. https://www.sciencedirect.com/science/article/pii/S2405457721001960.

Barazzoni, R., Bischoff, S. C., Breda, J., Wickramasinghe, K., Krznarić, Ž., Nitzan, D., \& Singer, P. (2020). ESPEN expert statements and practical guidance for nutritional management of individuals with SARS-CoV-2 infection. Liječnički vjesnik, 142(3-4), 75-84. https://covid19.elsevierpure.com/es/publications/espen-expert-statements-and-practical-guidance-for-nutritional-ma-2.

Brasil. Ministério da Saúde (2000). Agência Nacional de Vigilância Sanitária (ANVISA). Resolução - RDC no 63, de6 jul, 2000. http://bvsms.saude.gov.br/bvs/saudelegis/anvisa/2000/rdc0063_06_07_2000.html.

Doaei, S., Gholami, S., Rastgoo, S., Gholamalizadeh, M., Bourbour, F., Bagheri, S. E., \& Goodarzi, M. O. (2021). The effect of omega-3 fatty acid supplementation on clinical and biochemical parameters of critically ill patients with COVID-19: a randomized clinical trial. Journal of Translational Medicine, 19(1), 1-9. https://translational-medicine.biomedcentral.com/articles/10.1186/s12967-021-02795-5.

Farina, N., Nordbeck, S., Montgomery, M., Cordwin, L., Blair, F., Cherry-Bukowiec, J., \& Raymond, E. (2021). Nutrição enteral precoce em pacientes ventilados mecanicamente com infecção por COVID - $19 . \quad$ Nutrition in Clinical Practice, 36 (2), 440-448. https://aspenjournals.onlinelibrary.wiley.com/doi/10.1002/ncp.10629.

Ferreira, D. C., Santos, D. C., Vieira, M. P., Sampaio, N. D. C. S., \& de Andrade, S. S. (2020). Manejo nutricional para pacientes hospitalizados com COVID19: Uma revisão integrativa. Saúde Coletiva (Barueri), 10(59), 4140-4151. http://www.revistas.mpmcomunicacao.com.b r/index.php/saudecoletiva/article/view/1056.

Formenti, P., Bichi, F., Castagna, V., Pozzi, T., \& Chiumello, D. (2021). Nutrition Support in Patients With Acute Respiratory Distress Syndrome COVID-19. Nutrition in Clinical Practice. 36 (2), 500-501. https://pubmed.ncbi.nlm.nih.gov/33667020/.

Gonçalves, T. J. M., Gonçalves, S. E. A. B., Guarnieri, A., Risegato, R. C., Guimarães, M. P., de Freitas, D. C., \& Parrillo, E. F. (2021). Association Between Low Zinc Levels and Severity of Acute Respiratory Distress Syndrome by New Coronavirus SARS-CoV-2. Nutrition in Clinical Practice, 36(1), 186-191. https://aspenjournals-onlinelibrary-wiley.ez18.periodicos.capes.gov.br/doi/10.1002/ncp.10612.

Gorbalenya, A. E. et al. (2020). The species Severe acute respiratory syn-drome-related coronavirus: classifying 2019-nCoV and naming it SARS-CoV-2. Nature microbiology, 5(4), 536. https://www.researchgate.net/publication/339640926_The_species_Severe_acute_respiratory_syndromerelated_coronavirus_classifying_2019-nCoV_and_naming_it_SARS-CoV-2/link/5e5db512a6fdccbeba1468ea/download.

Gupta, L., Jalang'o, G. A., \& Gupta, P. (2020). Nutritional management and support in COVID-19: Emerging nutrivigilance. J Pak Med Assoc, 70(suppl 3), S124-S130. https://www.ejmanager.com/mnstemps/33/33-1589041587.pdf?t=1625835589.

Hoyois, A., Ballarin, A., Thomas, J., Lheureux, O., Preiser, J. C., Coppens, E., \& Arvanitakis, M. (2021). Nutrition evaluation and management of critically ill patients with COVID-19 during post-intensive care rehabilitation. Journal of Parenteral and Enteral Nutrition, 45(6), 1153-1163. https://aspenjournalsonlinelibrary-wiley.ez18.periodicos.capes.gov.br/doi/10.1002/jpen.2101.

Koekkoek, W. K., Panteleon, V., \& van Zanten, A. R. (2019). Current evidence on $\omega-3$ fatty acids in enteral nutrition in the critically ill: A systematic review and meta-analysis. Nutrition, 59, 56-68. https://pubmed.ncbi.nlm.nih.gov/30419501/.

Lechien, J. R., Chiesa-Estomba, C. M., De Siati, D. R., Horoi, M., Le Bon, S. D., Rodriguez, A., \& Saussez, S. (2020). Olfactory and gustatory dysfunctions as a clinical presentation of mild-to-moderate forms of the coronavirus disease (COVID-19): a multicenter European study. European Archives of Oto-RhinoLaryngology, 277(8), 2251-2261. https://pubmed.ncbi.nlm.nih.gov/32253535/.

Linko, R., Karlsson, S., Pettilä, V., Varpula, T., Okkonen, M., Lund, V., \& FINNALI Study Group*. (2011). Serum zinc in critically ill adult patients with acute respiratory failure. Acta Anaesthesiologica Scandinavica, 55(5), 615-621. https://pubmed.ncbi.nlm.nih.gov/21827444/.

Loris, P., Sasdelli, A. S., Ravaioli, F., Baracco, B., Battaiola, C., Bocedi, G., Brodosi, L. et al. (2021). Malnutrition and nutritional therapy in patients with SARS-CoV-2 disease. Clinical nutrition, 40(3), 1330-1337. https://pubmed.ncbi.nlm.nih.gov/32900518/. 10.1016/ j.clnu.2020.08.021.

Manzanares, W., Dhaliwal, R., Jurewitsch, B., Stapleton, R. D., Jeejeebhoy, K. N., \& Heyland, D. K. (2014). Parenteral fish oil lipid emulsions in the critically ill: a systematic review and meta-analysis. Journal of Parenteral and Enteral Nutrition, 38(1), 20-28. https://pubmed.ncbi.nlm.nih.gov/23609773/.

Martindale, R., Patel, J. J., Taylor, B., Arabi, Y. M., Warren, M., \& McClave, S. A. (2020). Nutrition therapy in critically ill patients with coronavirus disease 2019. Journal of parenteral and enteral nutrition, 44(7), 1174-1184. https://aspenjournals.onlinelibrary.wiley.com/doi/10.1002/jpen.1930.

McClave, S. A., Taylor, B. E., Martindale, R. G., Warren, M. M., Johnson, D. R., Braunschweig, C., \& Compher, C. (2016). Guidelines for the provision and assessment of nutrition support therapy in the adult critically ill patient: Society of Critical Care Medicine (SCCM) and American Society for Parenteral and Enteral Nutrition (ASPEN). JPEN. Journal of parenteral and enteral nutrition, 40(2), 159-211. https://pubmed.ncbi.nlm.nih.gov/26773077/.

Ministério da Saúde (Brasil). (2000). Resolução RDC nº 63, de 6 de julho de 2000: Aprova o Regulamento Técnico para fixar os requisitos mínimos exigidos para Terapia de Nutrição Enteral. http://bvsms.saude.gov.br/bvs/saudelegis/anvisa/2000/rdc0063_06_07_2000.html.

Moreira, E., Olano, E., \& Manzanares, W. (2020). Nutritional therapy in critically ill patients with COVID-19. A case review Terapia nutricional no paciente crítico com COVID-19. Uma revisão. Rev. méd. Urug, 102-130. http://www.scielo.edu.uy/scielo.php?script=sci_arttext\&pid=S1688-03902020000400102.

Osuna-Padilla, I., Rodríguez-Moguel, N. C., Aguilar-Vargas, A., \& Rodríguez-Llamazares, S. (2021). Safety and tolerance of enteral nutrition in COVID-19 critically ill patients, a retrospective study. Clinical Nutrition ESPEN, 43, 495-500. https://wwwsciencedirect.ez18.periodicos.capes.gov.br/science/article/pii/S240545772100084X?via\%3Dihub. 
Research, Society and Development, v. 10, n. 14, e412101422181, 2021

(CC BY 4.0) | ISSN 2525-3409 | DOI: http://dx.doi.org/10.33448/rsd-v10i14.22181

Patel, J. J., Martindale, R. G., \& McClave, S. A. (2020). Relevant nutrition therapy in COVID-19 and the constraints on its delivery by a unique disease process. Nutrition in Clinical Practice, 35(5), 792-799. https://www.ncbi.nlm.nih.gov/pmc/articles/PMC7436662/.

Pironi, L., Sasdelli, A. S., Ravaioli, F., Baracco, B., Battaiola, C., Bocedi, G., \& Musio, A. (2021). Malnutrition and nutritional therapy in patients with SARS-

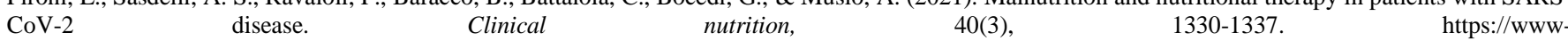
sciencedirect.ez18.periodicos.capes.gov.br/science/article/pii/S0261561420304374?via\%3Dihub.

Santos et al.. (2007). A estratégia PICO para a construção da pergunta de pesquisa e busca de evidências. Revista Latino-Americana de Enfermagem, 15 (3), 14. https://www.scielo.br/j/rlae/a/CfKNnz8mvSqVjZ37Z77pFsy/?lang=pt.

Silva, J. S., Espinheira, L. A. P., de Almeida, M. S., de Souza Ramos, M., Anunciação, S. M., Baptista, S. C. O., \& da Cunha, T. O. S. P. (2021). Terapia nutricional no paciente grave em cuidados paliativos. Medicina: Impactos Científicos e Sociais e Orientação a Problemas nas Diversas Áreas de Saúde, 1-388. https://www.atenaeditora.com.br/post-artigo/34896.

Singer, P., Blaser, A. R., Berger, M. M., Alhazzani, W., Calder, P. C., Casaer, M. P., \& Bischoff, S. C. (2019). ESPEN guideline on clinical nutrition in the intensive care unit. Clinical nutrition, 38(1), 48-79. https://pubmed.ncbi.nlm.nih.gov/30348463/1

Soares et al.. (2014). Revisão integrativa: conceitos e métodos utilizados na enfermagem. Revista da Escola de Enfermagem da USP, 48 (02), $335-345$. https://www.scielo.br/j/reeusp/a/3ZZqKB9pVhmMtCnsvVW5Zhc/?lang=pt.

Sousa, L. M. M., Marques-Vieira, C. M. A., Severino, S. S. P., \& Antunes, A. V. (2017). A metodologia de revisão integrativa da literatura em enfermagem. $\mathrm{N}^{\circ} 21$ Série 2-Novembro 2017, 17. https://www.researchgate.net/publication/321319742_Metodologia_de_Revisao_Integrativa_da_Literatura_ em_Enfermagem/link/5d7d2525a6fdcc2fof6fb0f9/download.

Souza, J. M. A. (2021). Terapia Nutricional No Paciente Grave Com Covid-19. Revista Científica Multidisciplinar Núcleo do Conhecimento, 05 (01), 120-130. https://www.nucleodoconhecimento.com.br/nutricao/paciente-grave.

Wu, Z., \& McGoogan, J. M. (2020). Characteristics of and important lessons from the coronavirus disease 2019 (COVID-19) outbreak in China: summary of a report of 72314 cases from the Chinese Center for Disease Control and Prevention. Jama, 323(13), 1239-1242. https://pubmed.ncbi.nlm.nih.gov/32091533/. 